\title{
USING DISTRIBUTED MAGNETOMETRY IN NAVIGATION OF HEAVY LAUNCHERS AND SPACE VEHICLES
}

N. Praly ${ }^{1}$, P.-J. Bristeau ${ }^{1}$, J. Laurent-Varin ${ }^{2}$, and N. Petit ${ }^{3}$

${ }^{1} \mathrm{SYSNAV}$

57 Rue de Montigny, Vernon 27200, France

${ }^{2}$ CNES Toulouse, CNES DCT/SI/GS

18 Av. Edouard Belin, Toulouse 31401, France

${ }^{3}$ MINES ParisTech, Centre Automatique et Systèmes

60 bd. Saint-Michel, Paris 75272, France

\begin{abstract}
Recently, a new technique (magneto-inertial navigation, MINAV) has emerged to address the general problem of reconstructing the inertial velocity of a rigid body moving in a magnetically disturbed region. The contribution of this paper is to apply the developed method, in a prospective spirit, to a case of space navigation in view of estimating the performance improvement that could be obtained using state-of-theart magnetometer technology onboard heavy launchers and other space vehicles. The main underlying idea of the approach is to estimate the inertial velocity by readings of the magnetic field at spatially distributed (known) locations on the rigid body. Mathematically, through a chainrule differentiation involving variables commonly appearing in classic inertial navigation, an estimate of this velocity can be obtained. This paper presents the potential of this method in the field of navigation of heavy launchers passing through particular regions of the Earth magnetosphere as considered, e.g., for upcoming Galileo missions. Numerical results based on the specifications of candidate embedded magnetic sensors stress the relevance of the approach. The presented methodology is patent pending and has been partially funded by CNES.
\end{abstract}

\section{INTRODUCTION}

The overall life expectation of a spacecraft is strongly related to the amount of fuel that is available onboard. Interestingly, the flight phase of injection into orbit of the payload is a critical phase with respect to this factor. The main reason for this is that the spacecraft may have to use a vast amount of its propellant to compensate for launchers position offsets. To minimize the undesired usage of 
propellants during this phase, a new generation of Ariane 5 launcher (Ariane 5 ME - Midlife Evolution) will be equipped with a reignitable engine: the Vinci rocket engine which can be used for the injection task. In this context, a new standard mission appears as very promising: the GTO+. It is a new version of the usual Geosynchronous Transfer Orbit (GTO) which is the historical mission of Ariane 5. The GTO apogee equals the altitude of the geosynchronous orbit, while its perigee lies between 200 and $250 \mathrm{~km}$ of altitude. The GTO+ has the same apogee, but its perigee is significantly higher (approximately $6000 \mathrm{~km}$ ). To reach this new orbit, the launcher trajectory includes a long coasting phase lasting approximately $5.5 \mathrm{~h}$. This very long phase is inconsistent with the accuracy of current inertial navigation systems, whose drift is proportional to the total flight time. Importantly, navigation devices are critical for the success of the mission as they are needed to estimate the (generalized) position of the launcher with respect to an inertial frame of reference. To maintain a good level of accuracy during such long-lasting launch missions, hybridizing the inertial measures with other sources of positioning information seems necessary. Unfortunately, classic technologies such as Global Navigation Satellite Systems (GNSS) or Global Positioning System (GPS) are discarded because they are easily blurred. In this paper, a new paradigm of navigation exploiting the Earth magnetic field to address this problem has been proposed. This technique, referred to as MINAV [1-3], and its extension to the field of space navigation are the subject of this paper.

The paper is organized as follows. In section 2, the principles of the MINAV technique for near-Earth application are exposed and a state-of-the-art on the useable magnetometers is reported. Section 3 gives a brief overview of the geomagnetic field model used to evaluate the spatial derivatives (gradient) of the magnetic field, which is one of the key factors for magneto-inertial navigation. In section 4, the potential gain of the MINAV is evaluated for a launcher during a GTO+ mission, and a method of hybridization is sketched.

\section{MAGNETO-INERTIAL NAVIGATION}

\subsection{Notation and Reference Frames}

The following frames of reference are considered:

- the inertial Earth frame (index 'if') which has its origin at the center of the Earth. Its axes are not rotating with the Earth. The $O z_{\text {if }}$ axis coincides with the Earth's polar axis (which is assumed to have a constant direction);

- the Earth rotating frame (index 'Ef') which has its origin at the center of the Earth. Its axes are attached to the Earth. The $O z_{\mathrm{Ef}}$ axis coincides with the Earth's polar axis; and 
Table 1 Nomenclature and notations (IGRF - International Geomagnetic Reference Field)

\begin{tabular}{|c|c|c|}
\hline Symbol & Quantity & Unit \\
\hline$\vec{B}$ & Magnetic field vector & $\mathrm{T}$ or $\mathrm{G}$ \\
\hline$\vec{X}$ & Position of the rigid body & $\mathrm{m}$ \\
\hline$\frac{\partial B}{\partial X}$ & Spatial derivatives (Jacobian) of the magnetic field & $\mathrm{T} / \mathrm{m}$ or $\mathrm{G} / \mathrm{m}$ \\
\hline$M_{\mathrm{if} \rightarrow \mathrm{bf}}$ & Matrix transformation from inertial frame to body frame & - \\
\hline$\vec{\Omega}_{\mathrm{if} \rightarrow \mathrm{bf}}$ & Angular speed from inertial frame to body frame & $\mathrm{rad} / \mathrm{s}$ \\
\hline $\overrightarrow{A^{\mathrm{bf}}}$ & Vector $A$ written in the body frame axis set & $|A|$ \\
\hline$\left.\frac{d \vec{A}}{d t}\right|_{\mathrm{bf}}$ & Time derivative of the vector $A$ in the body frame & $|A| / \mathrm{s}$ \\
\hline$\left.\frac{d \vec{A}^{\mathrm{if}}}{d t}\right|_{\mathrm{bf}}$ & $\begin{array}{l}\text { Time derivative of the vector } A \text { in the body frame, } \\
\text { expressed in the inertial frame axis set }\end{array}$ & $|A| / \mathrm{s}$ \\
\hline$\Delta \vec{A}$ & First-order error on the component of $A$ & $|A|$ \\
\hline$V(R, \theta, \phi)$ & Magnetic potential (IGRF model) & $\mathrm{T} \cdot \mathrm{m}$ or $\mathrm{G} \cdot \mathrm{m}$ \\
\hline$a$ & Mean Earth's radius (IGRF model) & $\mathrm{m}$ \\
\hline$R$ & Radius & $\mathrm{m}$ \\
\hline$\theta$ & Colatitude (IGRF model) & $\mathrm{rad}$ \\
\hline$\phi$ & Longitude (IGRF model) & $\mathrm{rad}$ \\
\hline$g_{n, m}, h_{n, m}$ & IGRF-2011 coefficients & \\
\hline$P_{n, m}$ & Legendre polynomials & \\
\hline
\end{tabular}

- the body frame (index 'bf') which is fixed to the body under consideration (vehicle). The orthogonal axes are aligned with the roll, pitch, and yaw axes of the vehicle, respectively.

Notations are reported in Table 1.

\subsection{Basic Equations of Magneto-Inertial Navigation}

Magneto-inertial navigation is a newly introduced technique to estimate the velocity of a rigid body in a spatially disturbed and time-invariant magnetic field [1$3]$. It has been introduced for ground navigation applications (for pedestrians [3, 4] and automotive vehicles [5,6], mostly). Interestingly, it can be generalized to space vehicles, at the expense of more sophisticated equations modeling the Earth magnetic field as rotating with the Earth. As will be discussed, MINAV uses inertial measurements to evaluate some parameters. Therefore, the body under consideration is assumed to be equipped with an Inertial Measurement Unit (IMU). 
The main equation used is the chain-rule differentiation of the sensed magnetic field:

$$
\frac{d \vec{B}}{d t}=\frac{\partial \vec{B}}{\partial t}+\left[\frac{\partial B}{\partial X}\right] \frac{d \vec{X}}{d t} .
$$

Let now detail each term in Eq. (1):

- $d \vec{B} / d t$ can be measured with a 3 -axes magnetometer attached to the body. The measure is $d \vec{B} /\left.d t\right|_{\mathrm{bf}}$;

- $\partial \vec{B} / \partial t$ cannot be easily measured. The following assumption is made: at the vicinity of the Earth $(<800 \mathrm{~km})$, the magnetic field is rotating with the Earth. Therefore, $\partial \vec{B} /\left.\partial t\right|_{\mathrm{Ef}}=\overrightarrow{0}$;

- $[\partial B / \partial X]$ is the magnetic field gradient. This quantity can be estimated from measurements obtained with an array of 3-axes magnetometers located at known distinct locations in the body; and

- $d \vec{X} / d t$ is the body velocity with respect to inertial Earth frame. To use this variable in the navigation algorithms, one needs to consider $d \vec{X} /\left.d t\right|_{\text {if }}$.

Considering the above measurement principles, let express the vector equation (1) in the Earth rotating frame:

$$
\left.\frac{d \vec{B}}{d t}\right|_{\mathrm{Ef}}=\left.\frac{\partial \vec{B}}{\partial t}\right|_{\mathrm{Ef}}+\left.\left[\frac{\partial B}{\partial X}\right] \frac{d \vec{X}}{d t}\right|_{\mathrm{Ef}} .
$$

This equation is transformed under the following form to make the velocity with respect to the inertial Earth frame appear

$$
\left.\frac{d \vec{B}}{d t}\right|_{\mathrm{Ef}}=\left.\frac{\partial \vec{B}}{\partial t}\right|_{\mathrm{Ef}}+\left[\frac{\partial B}{\partial X}\right]\left(\left.\frac{d \vec{X}}{d t}\right|_{\mathrm{if}}+\vec{\Omega}_{\mathrm{if} \rightarrow \mathrm{Ef}} \otimes \vec{X}\right) .
$$

As one can measure $d \vec{B} /\left.d t\right|_{\mathrm{bf}}$, one can use the following transformation:

$$
\left.\frac{d \vec{B}}{d t}\right|_{\mathrm{Ef}}=\left.\frac{d \vec{B}}{d t}\right|_{\mathrm{bf}}+\vec{\Omega}_{\mathrm{bf} \rightarrow \mathrm{Ef}} \otimes \vec{B}
$$

in Eq. (2) and finally obtain:

$$
\left.\frac{d \vec{B}}{d t}\right|_{\mathrm{bf}}=-\vec{\Omega}_{\mathrm{bf} \rightarrow \mathrm{Ef}} \otimes \vec{B}+\left[\frac{\partial B}{\partial X}\right]\left(\left.\frac{d \vec{X}}{d t}\right|_{\mathrm{if}}+\vec{\Omega}_{\mathrm{if} \rightarrow \mathrm{Ef}} \otimes \vec{X}\right) .
$$

Let now discuss each terms appearing in Eq. (3): 
$-\vec{\Omega}_{\mathrm{bf} \rightarrow \text { Ef }}$ is the sum of $\vec{\Omega}_{\mathrm{bf} \rightarrow \text { if }}$ which is the angular velocity of the body with respect to inertial Earth frame (measured with a 3-axes gyrometer) and $\vec{\Omega}_{\text {if } \rightarrow \text { Ef }}$ which is the angular velocity of the Earth with respect to inertial Earth frame; and

- $\vec{X}$ is the position of the body and can be determined by integrating the acceleration (measured by the IMU) or the velocity.

Because all the magnetic measurements are performed in the body frame, let now express Eq. (3) in this reference frame:

$$
\begin{aligned}
\left.\frac{d \vec{B}^{\mathrm{bf}}}{d t}\right|_{\mathrm{bf}}= & -\vec{\Omega}_{\mathrm{bf} \rightarrow \mathrm{if}}^{\mathrm{bf}} \otimes \vec{B}^{\mathrm{bf}}-\left(M_{\mathrm{if} \rightarrow \mathrm{bf}} \vec{\Omega}_{\mathrm{if} \rightarrow \mathrm{Ef}}^{\mathrm{if}}\right) \otimes \vec{B}^{\mathrm{bf}} \\
& +\left.\left[\frac{\partial B}{\partial X}\right]^{\mathrm{bf}} M_{\mathrm{if} \rightarrow \mathrm{bf}} \frac{d \vec{X}^{\mathrm{if}}}{d t}\right|_{\mathrm{if}}+\left[\frac{\partial B}{\partial X}\right]^{\mathrm{bf}} M_{\mathrm{if} \rightarrow \mathrm{bf}}\left(\vec{\Omega}_{\mathrm{if} \rightarrow \mathrm{Ef}}^{\mathrm{if}} \otimes \vec{X}^{\mathrm{if}}\right) .
\end{aligned}
$$

To evaluate the potential accuracy improvement obtained by hybridization of the inertial navigation and the magneto-inertial technique, the accuracy of the standard IMU and the accuracy of embeddable magnetic sensors will be detailed. With this aim in mind, the data from the Ariane 5 IMU are used and analyzed with regard to Eq. (4). Preliminary to this analysis, a state-of-the-art of magnetometers is presented in the following subsection.

\subsection{Magnetometers State-of-the-Art}

Various sensing technologies could be embedded onboard a heavy launcher such as Ariane 5. Because it is necessary to evaluate the three coordinates of the magnetic field, only vector magnetometers are considered in the study. Table 2 reports a list of the main technologies along with their respective advantages and drawbacks [7].

Ssuperconducting quantum interference device (SQUID) sensors seem to be the only available technology measuring the magnetic field with enough accuracy to exploit the principles of MINAV in space applications.

To evaluate the magnetic field gradient, an array of 3-axes magnetometers is used. Two configurations can be considered:

(1) a tight network of magnetometers will ensure a thermal stability and an increased knowledge of the mechanical properties (distance between the magnetometers, orientation, limited mechanical deformation, etc.). On the other hand, the sensitivity of this arrangement is greatly reduced; and

(2) a spread-out network of magnetometers will ensure a greater sensitivity, but thermal and mechanical fluctuations may reveal much larger than in the compact network. 
Table 2 Magnetometers state-of-the-art (GMR — giant magnetoresistance; MTJ magnetic tunnel junction)

\begin{tabular}{lllc}
\hline \multicolumn{1}{c}{ Technology } & \multicolumn{1}{c}{ Advantages } & \multicolumn{1}{c}{ Drawbacks } & $\begin{array}{c}\text { Accuracy, } \\
\mathrm{nT}\end{array}$ \\
\hline Fluxgate* & $\begin{array}{l}\text { Good accuracy; } \\
\text { most exploited } \\
\text { technology } \\
\text { in space applications }\end{array}$ & Creates a magnetic field & $10^{-2}-10^{7}$ \\
\hline GMR sensors & Small size & Poor accuracy & $10^{1}-10^{8}$ \\
\hline $\begin{array}{l}\text { Magnetoelectric } \\
\text { sensors }\end{array}$ & $\begin{array}{l}\text { Good accuracy; } \\
\text { small size }\end{array}$ & $\begin{array}{l}\text { Nonlinearity for measuring } \\
\text { small magnetic field }\end{array}$ & $10^{-3}-10^{8}$ \\
\hline MTJ sensors & Small size & Poor accuracy & $10^{1}-10^{8}$ \\
\hline Search-coil* & Very good accuracy & Time-dependent magnetic field & $10^{-5}-10^{8}$ \\
\hline SQUID sensors & $\begin{array}{l}\text { Best accuracy; } \\
\text { low noise }\end{array}$ & Low working temperature & $10^{-6}-10^{0}$ \\
\hline${ }^{*}$ Already used in & space applications. & &
\end{tabular}

${ }^{*}$ Already used in space applications.

\section{MODEL OF EARTH MAGNETIC FIELD AND EVALUATION OF ITS GRADIENT}

Numerous models of the Earth magnetic field have been developed (see, e. g., [810]). Earlier, the assumption of a time-invariant magnetic field in the Earth frame at low altitudes has been formulated. An internal-source field model is consistent with the hypothesis. Among such models is the IGRF-2011 [10]. This model describes the geomagnetic internal field and its secular variations, as being the gradient of a scalar potential $V$ which is expressed through a spherical harmonic expansion with respect to spherical geocentric coordinates:

$$
\begin{aligned}
& V(R, \theta, \phi) \\
& \quad=a \sum_{n=1}^{N} \sum_{m=0}^{n}\left(\frac{a}{R}\right)^{n+1}\left(g_{n, m} \cos (m \phi)+h_{n, m} \sin (m \phi)\right) P_{n, m}(\cos (\theta)) .
\end{aligned}
$$

Notations are given in Table 1.

The potential model (5) can be used to evaluate the spatial derivatives of the magnetic field at any point. In Fig. 1, the variations of the components of the magnetic field gradient are reported for altitude variations typical of a launch vehicle performing a GTO mission (from liftoff to geostationary Earth orbit (GEO) altitude). A typical value of $10^{-2}$ to $10^{-3} \mathrm{nT} / \mathrm{m}$ is observed for 
altitudes below $800 \mathrm{~km}$. Such values can be effectively measured with an embedded network of SQUID sensors located at $1 \mathrm{~m}$ from each other, because their theoretical sensitivity is $10^{-6} \mathrm{nT} / \mathrm{m}$. Interestingly, thanks to this high sensitivity, the measure of the magnetic field gradient could also be achieved at GEO altitude. However, at this altitude, the assumption of a time-invariant magnetic field in the Earth frame may prove untrue for multiple reasons, e. g., solar activity.

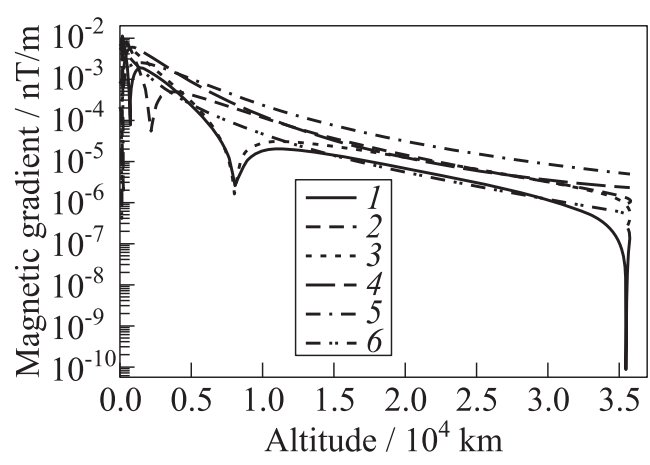

Figure 1 Variations of some components of the magnetic field Jacobian along a GTO launch mission: $1-\partial B_{x} / \partial X ; 2-\partial B_{x} / \partial Y ; 3-\partial B_{x} / \partial Z$; $4-\partial B_{y} / \partial Y ; 5-\partial B_{y} / \partial Z ;$ and $6-\partial B_{z} / \partial Z$

\section{PERFORMANCE OF MAGNETO-INERTIAL NAVIGATION DURING A GEOSYNCHRONOUS TRANSFER ORBIT MISSION}

\subsection{Accuracy}

Equation (4) is the projection of Eq. (3) onto the body frame axes set. Let now estimate the propagation of errors (uncertainties) in this equation. Using a current Ariane 5 IMU, the orientation error is very small $\left(\approx 10^{-8}\right.$ rad after 1500 s). Developing Eq. (3) at first order gives:

$$
\begin{aligned}
&\left.\Delta \frac{d \vec{B}}{d t}\right|_{\mathrm{bf}}=-\Delta \vec{\Omega}_{\mathrm{bf} \rightarrow \mathrm{if}} \otimes \vec{B}-\vec{\Omega}_{\mathrm{bf} \rightarrow \mathrm{if}} \otimes \Delta \vec{B}-\vec{\Omega}_{\mathrm{if} \rightarrow \mathrm{Ef}} \otimes \Delta \vec{B} \\
&+\Delta\left[\frac{\partial B}{\partial X}\right]\left(\left.\frac{d \vec{X}}{d t}\right|_{\mathrm{if}}+\vec{\Omega}_{\mathrm{if} \rightarrow \mathrm{Ef}} \otimes \vec{X}\right) \\
&+\left[\frac{\partial B}{\partial X}\right]\left(\left.\Delta \frac{d \vec{X}}{d t}\right|_{\mathrm{if}}+\vec{\Omega}_{\mathrm{if} \rightarrow \mathrm{Ef}} \otimes \Delta \vec{X}\right) .
\end{aligned}
$$

An estimation of the accuracy for a GTO injection point $(\approx 700 \mathrm{~km}$ from Earth) is given in Table 3 using Eq. (6) and the sensors accuracies. The Ariane 5 IMU bias performance is below $1 \mathrm{mg}$ [11]. Therefore, on a typical GTO mission, 
Table 3 Accuracy of magnetic navigation at an actual injection point

\begin{tabular}{ccc}
\hline Parameter & Order of magnitude & Unit \\
\hline$\left.\Delta \frac{d \vec{B}}{d t}\right|_{\mathrm{bf}}$ & $10^{-6}$ & $\mathrm{nT} / \mathrm{s}$ \\
$\Delta \vec{B}$ & $10^{-6}$ & $\mathrm{nT}$ \\
$\Delta\left[\frac{\partial B}{\partial X}\right]$ & $10^{-6}$ & $\mathrm{nT} / \mathrm{m}$ \\
$\Delta \vec{\Omega}_{\mathrm{bf} \rightarrow \text { if }}$ & $10^{-8 *}$ & $\mathrm{rad} / \mathrm{s}$ \\
$\Delta \vec{X}$ & $10^{3 * *}$ & $\mathrm{~m}$ \\
$\vec{B}$ & $10^{4}$ & $\mathrm{nT}$ \\
{$\left[\frac{\partial B}{\partial X}\right]$} & $10^{-2}-10^{-3}$ & $\mathrm{nT} / \mathrm{m}$ \\
$\left.\frac{d \vec{X}}{d t}\right|_{\text {if }}$ & $10^{3}$ & $\mathrm{~m} / \mathrm{s}$ \\
$\overrightarrow{\Omega_{\mathrm{bf} \rightarrow \text { if }}}$ & $10^{-2}$ & $\mathrm{rad} / \mathrm{s}$ \\
$\vec{\Omega}_{\mathrm{if} \rightarrow \text { Ef }}$ & $10^{-5}$ & $\mathrm{rad} / \mathrm{s}$ \\
$\vec{X}$ & $10^{6}$ & $\mathrm{~m}$ \\
$\left.\Delta \frac{d \vec{X}}{d t}\right|_{\text {if }}$ & $10^{0}-10^{-1}$ & $\mathrm{~m} / \mathrm{s}$ \\
\hline${ }^{*}$ IMU parameter (from [11]). \\
${ }^{* *}$ Injection accuracy (from [12]). &
\end{tabular}

the velocity error generated by the inertial navigation system, at the injection point, is around $10 \mathrm{~m} / \mathrm{s}$. Considering the data reported in Table 3, one can conclude that MINAV has the potential to improve the injection accuracy by a factor of 10 to 100 .

\subsection{Typical Use of Magneto-Inertial Navigation in Launch Vehicle and Other Spacecraft}

By contrast to pure inertial systems, MINAV devices can provide an absolute estimate of velocity (which may be uncertain but does not time-drift as it is not the result of an integration over time). As long as the orders of magnitude of the various parameters remain unchanged, the accuracy on the estimated velocity is constant. Yet, during the launch mission, the magnetic field gradient decreases as discussed earlier and reported in Fig. 1. Then, the obtained velocity estimate becomes less and less accurate. With respect to the preceding discussion, one can define a 3-phase navigation strategy for a launch mission. This strategy is illustrated in Fig. 2. It combines traditional inertial navigation and magnetoinertial techniques: 


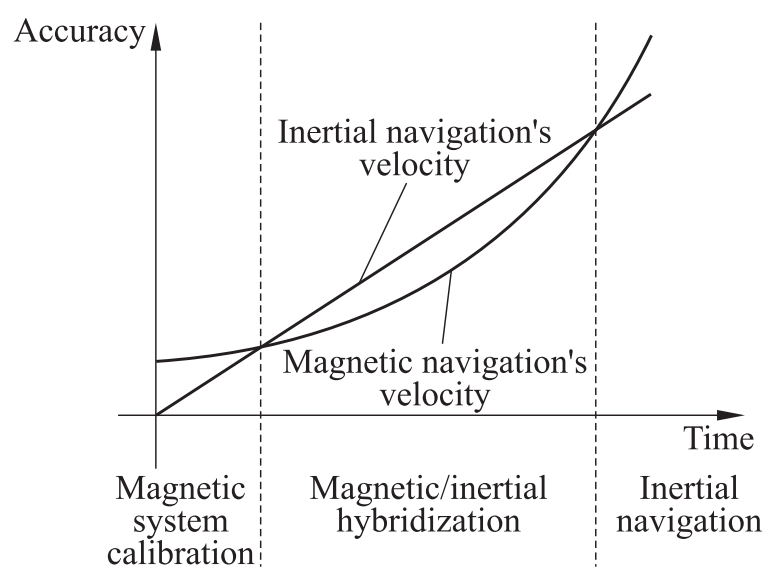

Figure 2 Schematic view of drifts of inertial and magneto-inertial navigation during a space launch

(1) the first phase consists in using the inertial navigation estimation to calibrate the MINAV system. During this phase, the inertial navigation is accurate as the drift is small;

(2) after some time, due to the inertial navigation drift, the MINAV technique provides a better estimate of the velocity than the inertial system. Hybridization of the inertial and magneto-inertial information yields an improvement of the flight parameter estimates; and

(3) as the altitude rises, the MINAV velocity estimates loses accuracy. Then, the inertial navigation technique, whose error has been kept small during the previous phase (second phase), is used for the rest of the mission.

\section{CONCLUDING REMARKS}

Information about absolute and relative to the Earth velocity is critical for numerous space applications including payload injection into orbit. The MINAV technique presented in this paper proposes a new solution to this problem. Considering the theoretical performance of SQUID magnetometers, it has been shown that this technology has the potential of improving the current launch vehicle navigation accuracy (and, thus, the injection accuracy) by a factor of 10 to 100 .

The potential gain is related to $(i)$ the magnitude of the magnetic field gradient observed along the trajectory; and $(i i)$ the accuracy of the employed magnetometers. This preliminary study and its promising results call for further investigations. Information on the real near-Earth magnetic gradient and the 
impact of the space vehicle electrical environment onto the magnetometers sensors array are critical to evaluate the actually obtainable performance.

Finally, it is interesting to note that other potential applications for MINAV can be found in the domain of interplanetary space probe navigation. As MINAV takes advantage of the disturbances of the magnetic field, every celestial body, with a sufficiently strong and stationary magnetic field and a known motion, could allow to compensate the drift of classic inertial navigation systems.

\section{REFERENCES}

1. Vissière, D., A.P. Martin, and N. Petit. 2007. Système fournissant vitesse et position d'un corps en utilisant les variations du champ magnétique évaluées grâce aux mesures de un ou des magnétomètres et de une ou des centrales inertielles. SYSNAV Patent No. 0702431.

2. Vissière, D., A.P. Martin, and N. Petit. 2007. Using magnetic disturbances to improve IMU-based position estimation. European Control Conference Proceedings.

3. Dorveaux, E. 2011. Magneto-inertial navigation: Principles and application to an indoor pedometer. Ph.D. Thesis. Ecole Nationale Supérieure des Mines de Paris.

4. Dorveaux, E., T. Boudot, M. Hillion, and N. Petit. 2011. Combining inertial measurements and distributed magnetometry for motion estimation. American Control Conference Proceedings.

5. Bristeau, P.-J., and N. Petit. 2011. Navigation system for ground vehicles using temporally interconnected observers. American Control Conference Proceedings.

6. Bristeau, P.-J. 2011. Motion estimation techniques for GPS-free vehicle and other examples of MEMS navigation systems design. Ph.D. Thesis. Ecole Nationale Supérieure des Mines de Paris.

7. Lenz, J., and A. S. Edelstein. 2006. Magnetic sensors and their applications. IEEE Sensors J. 6(3):631-49.

8. Olson, W. P., and K. A. Pfitzer. 1974. Quantitative model of the magnetospheric magnetic field. J. Geophys. Res. 79:3739.

9. Tsyganenko, N. A. 2002. A model of the near magnetosphere with a dawn-dusk asymmetry 1. J. Geophys. Res. 107(A8).

10. International Association of Geomagnetism and Aeronomy, Working Group V-MOD. 2010. International geomagnetic reference field: The eleventh generation. Int. Geophys. J. 183:1216-30.

11. Broquet, R., N. Perrimon, B. Polle, et al. 2010. HiNAV Inertial/GNSS hybrid navigation system for launchers and re-entry vehicles. Satellite Navigation Technologies and European Workshop on GNSS Signals and Signal Processing (NAVITEC). 5th ESA Workshop. DOI:10.1109/NAVITEC.2010.5707998.

12. Ariane 5. User's Manual. July 2008. Issue 5. Revision 0. 